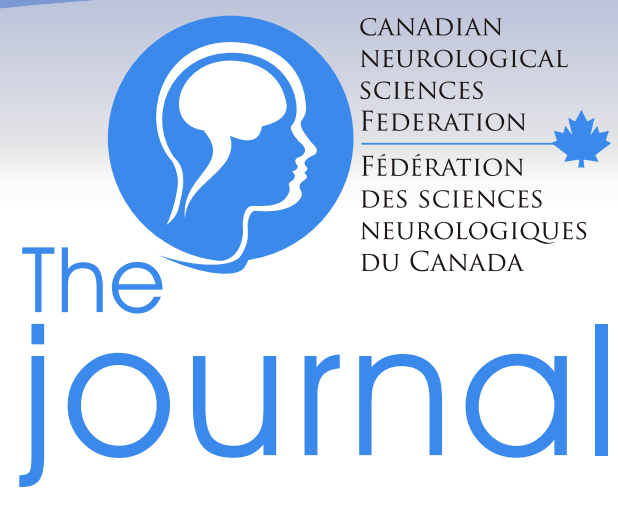

Canadian Journal of Neurological Sciences

Volume $44 \quad$ (Supplement 2) June 2017

\title{
52nd Annual Congress of the \\ Canadian Neurological \\ Sciences Federation
}

Victoria, British Columbia, Canada

52e congrès annuel de la

Fédération des sciences

neurologiques du Canada

\section{ABSTRACTS / RÉSUMÉS}




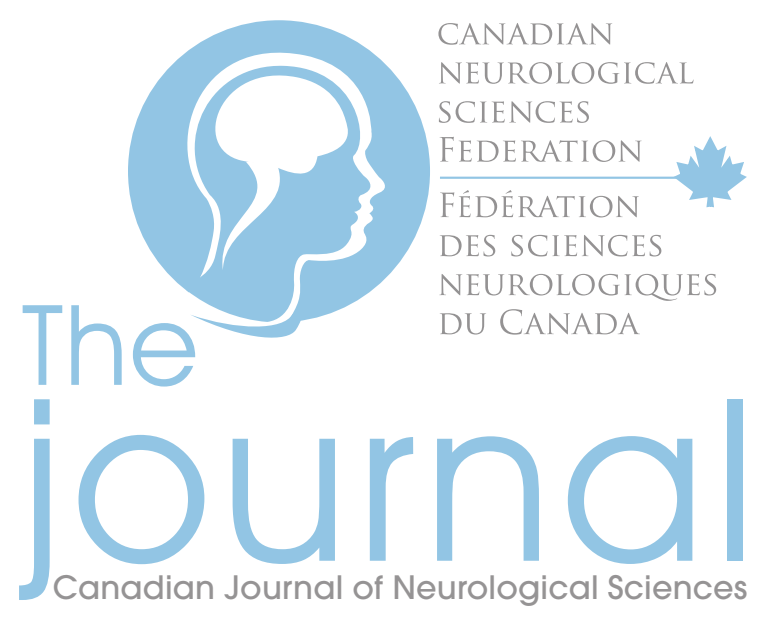

\section{REVIEWER OF THE YEAR - HARVEY B. SARNAT, M.S., M.D., F.R.C.P.C.}

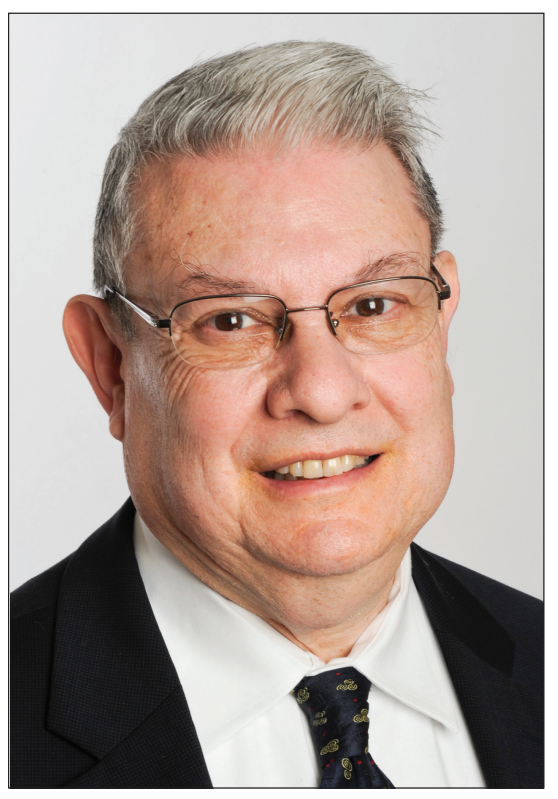

Harvey B. Sarnat is Professor of Paediatrics, Pathology (Neuropathology) and Clinical Neurosciences at the University of Calgary and Alberta Children's Hospital in Calgary, Canada since 1981, except for a decade in which he returned to the U.S. to serve on faculty at the University of Washington (Seattle) and later UCLA (Los Angeles). He began his career at the University of Illinois, earning a B.S. degree in zoology before entering the U. of I. College of Medicine, Chicago, in a dual programme, earning an M.S. degree in neuroanatomy a year before his M.D. degree in 1966. After medical school he remained at the U. of I. Hospitals in Chicago to perform a residency in Paediatrics, later followed by residencies in Paediatric Neurology and Neuropathology at the University of Virginia in Charlottesville. Research interests include neuroembryology, developmental/fetal neuropathology, epilepsy neuropathology, congenital myopathies and mitochondrial cytopathies. Publications include 190 original peer-reviewed research articles and 100 books and book chapters, mostly on these topics. He sits on Editorial Boards of 9 journals and is an ad hoc reviewer for several others. From 1984-1992 he served as Associate Editor of the Canadian Journal of Neurological Sciences. In 2013 an annual endowed Harvey Sarnat Developmental Neuroanatomy and Neuropathology Lectureship was established at Alberta Children's Hospital in his honour. He is one of 8 members of the Neuropathology Commission of the International League Against Epilepsy. He was honoured as the 2010 Gordon Matheson Lecturer at the 50 th anniversary meeting of the Canadian Association of Neuropathologists, and as the prestigious Bernard Sachs Lecturer at the 2016 meeting of the (USA) Child Neurology Society. His principal research interests are in developmental neuroanatomy (neuroembryology) and neuropathology; epilepsy neuropathology; and phylogenetic (evolutionary) neurobiology. 


\title{
52nd Annual Congress of the \\ Canadian Neurological Sciences Federation
}

British Columbia, Victoria • JUNE 20 - 23, 2017

\section{CNSF EXecutive CoMmitTeE}

Jeanne Teitelbaum, CNSF/NSFC President

Kesh Reddy, CNSF/NSFC Vice President

Michael Hill, CNSF/NSFC Vice President

Sharon Whiting, CNSF/NSFC Vice President

J. Max Findlay CNSF/NSFC Past President

Dan Morin, CNSF/NSFC Board Member - CNSF Chief Executive Officer

Juliette Hukin, CNSF/NSFC Board Member - CACN President Cecil Hahn, CNSF/NSFC Board Member - CACN Vice President Stephen Lownie, CNSF/NSFC Board Member - CNSS President Pat McDonald CNSF/NSFC Board Member - CNSS Vice President Colin Chalk, CNSF/NSFC Board Member - CNS President Fionna Costello, CNSF/NSFC Board Member - CNS Vice President Jose Francisco Tellez-Zenteno, CNSF/NSFC Board Member - CSCN President Fraser Moore, CNSF/NSFC Board Member - CSCN Vice President Bill Wang, CNSF/NSFC Board Member - CNSS Resident Representative Theo Mobach, CNSF/NSFC Board Member - CNS Resident Representative Leyila Kaseka, CNSF/NSFC Board Member - CACN Resident Representative Ian Fleetwood CNSF/NSFC Board Member - CNSF At-large Kristine Chapman, CNSF/NSFC Board Member - CNSF At-large Robert Chen, CJNS Editor-in-Chief-CNS + CSCN Member Tejas Sankar, CNSF SPC Chair Joe Megyesi, CNSF PDC Chair Richard Riopelle, CNSF Advocacy Chair - CNS Member Shobhan Vachhrajani, CNSF CPGC Chair-CNSS Member

\author{
SCIENTIFIC PROGRAM COMMITTEE \& \\ PROFESSIONAL DEVELOPMENT COMMITTEE \\ Joe Megyesi \\ Aleksandra Mineyko \\ Tejas Sankar \\ PDC Chair (CNSS) \\ Alexandre Henri-Bhargava \\ Chintankumar Shah \\ Shannon Venance \\ Craig Campbell \\ Michael Esser \\ Phillippe Couillard \\ Gelareh Zadeh \\ Danielle Andrade \\ Seyed Mirsattari \\ Leyila Kaseka \\ Theo Mobach \\ Bill Wang \\ Mark Bigder \\ Jeanne Teitelbaum \\ Kesh Reddy \\ Sharon Whiting \\ $P D C$ Vice-Chair (CACN) \\ SPC Chair (CNSS) \\ $S P C$ Vice-Chair $(C N S+C S C N)$ \\ $P D C(C A C N)$ \\ $P D C(C S C N)$ \\ $S P C(C A C N+C S C N)$ \\ $S P C(C A C N)$ \\ $S P C(C N S)$ \\ $S P C$ (CNSS) \\ $S P C(C S C N)$ \\ $S P C(C S C N)$ \\ $S P C+P D C$ Residents Rep $(C A C N)$ \\ $S P C+P D C$ Residents Rep (CNS) \\ $S P C+P D C$ Residents Rep (CNSS) \\ $S P C+P D C$ Junior Residents Rep (CNSS) \\ CNSF/NSFC President (CNS) \\ CNSF/NSFC Vice President (CNSS) \\ CNSF/NSFC Vice President $(C A C N+C S C N)$
}

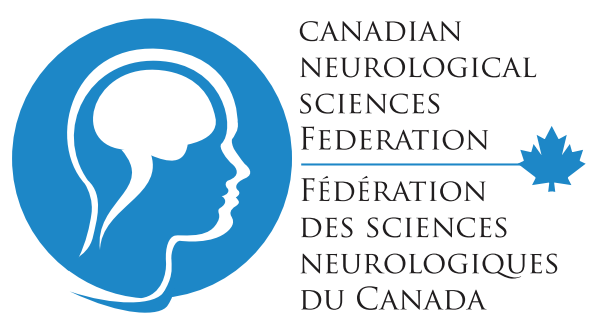

\section{ABSTRACTS AND PROGRAM}

CANADIAN NEUROLOGICAL SCIENCES FEDERATION

143N - 8500 Macleod Trail SE

Calgary, Alberta T2H 2N1 Canada

Tel: (403) 229-9544 • Fax: (403) 229-1661 


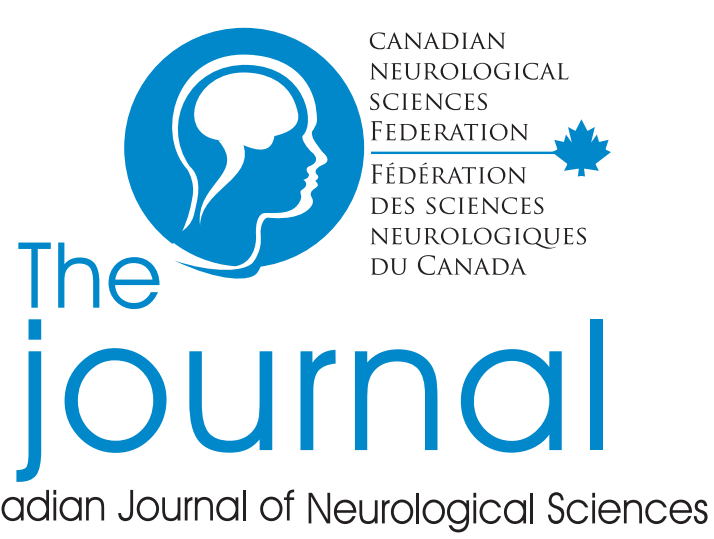

\section{Volume 44 / Supplement 2 / June 2017}

\section{Editor-in-Chief/Rédactueur en chef}

Robert Chen TORONTO, ON

\section{Associate Editors/Rédacteurs associés}

Etienne De Villers-Sidani MONTREAL, QC

Robert Hammond LONDON, ON

Hans Katzberg TORONTO, ON

Mahendranath Moharir TORONTO, ON

Tejas Sankar EDMONTON, AB

Manas Sharma LONDON, ON

Jeanne Teitelbaum MONTREAL, QC

\section{Past Editors/Anciens rédacteurs en chef}

G. Bryan Young LONDON, ON Douglas W. Zochodne CALGARY, AB James A. Sharpe TORONTO, ON Robert G. Lee CALGARY, AB Robert T. Ross WINNIPEG, MB (Emeritus Editor, Founding Editor)

\section{Editorial Board/Comité éditorial}

Jorge Burneo, LONDON, ON Jodie Burton, CALGARY, AB Colin Chalk, MONTREAL, QC K. Ming Chan, EDMONTON, AB Alan Goodridge, ST. JOHN's, NL Mark Hamilton, CALGARY, AB Michael Hill, CALGARY, AB Alan C. Jackson, WINNIPEG, MB Draga Jichici, HAMILTON, ON Suneil Kalia, TORONTO, ON Daniel Keene, OTTOWA, ON Julia Keith, TORONTO, ON Stephen Lownie, LONDON, ON Jian-Qiang Lu, EDMONTON, AB Patrick McDonald, vancouver, BC Joseph Megyesi, LONDON, ON
Tiago Mestre, OTTOWA, ON Sarah Morrow, LONDON, ON Michael Nicolle, LONDON, ON Narayan Prasad, LONDON, ON Alex Rajput, SASKATOON, SK Kesh Reddy, HAMILTON, ON Ramesh Sahpaul, NORTH VANCOUVER, BC Dipanka Sarma, TORONTO, ON Sean Symons, TORONTO, ON Brian Toyota, vancouver, BC Brian Weinshenker, ROCHESTER, MN Sam Wiebe, CALGARY, AB Eugene $\mathrm{Yu}$, TORONTO, ON

\section{Book Review/Critiques de livres Reflections/Reflets}

Andrew Kirk SASKATOON, SK

\section{Journal Staff/Effectif du Journal}

Dan Morin CALGaRY, AB

Chief Executive Officer

Donna Irvin CALGARY, AB

CNSF Membership Services /

Communications Officer

\section{The official journal of: / La Revue officielle de:}

The Canadian Neurological Society La Société Canadienne de Neurologie

The Canadian Neurosurgical Society La Société Canadienne de Neurochirurgie

The Canadian Society of Clinical Neurophysiologists La Société Canadienne de Neurophysiologie Clinique

The Canadian Association of Child Neurology L' Association Canadienne de Neurologie Pédiatrique

The permanent secretariat for the four societies and the Canadian Neurological Sciences Federation is at:
Le sécretariat des quatre associations et de la Fédération des sciences neurologiques du Canada est situe en permanence à :

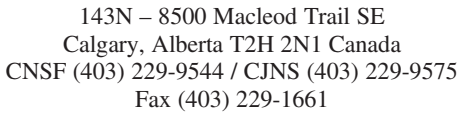

The Canadian Journal of Neurological Sciences is published bi-monthly. The annual subscription rate for Individuals (electronic) is $£ 125 / \$ 207$. The annual subscription rate for Institutions (electronic) is $£ 167 / \$ 277$. See <cambridge.org/cjn > for full details including taxes; e-mail: subscriptions newyork@cambridge.org. The Canadian Journal of Neurological Sciences is included in the Cambridge Core service, which can be accessed at cambridge.org/ cjn. For information on other Cambridge titles, visit www.cambridge.org. For advertising rates contact M. J. Mrvica Associates, 2 West Taunton Avenue, Berlin, NJ 08009; Phone: 856-768-9360; Fax: 856-753-0064; Email mjmrvica@mrvica.com.

Le Journal Canadien des Sciences Neuorlogiques est publié tous les deux mois. Le prix d'abonnement annuel pour les individus (électronique) est 125£/207\$. Le prix d'abonnement annuel pour les établissements (électronique) est $167 £ / 277 \$$. Veuillez consulter <cambridge.org/cjn> pour tous les détails, y compris les taxes; email: subscriptions_newyork@cambridge.org. Le Journal canadien des sciences neurologiques est inclus dans le service Cambridge Journals Online, accessible à cambridge.org/cjn. Pour plus d'informations sur les titres disponible chez Cambridge, veuillez consulter www. cambridge.org. Pour les tarifs de publicité, contacter M. J. Mrvica Associates, 2 West Taunton Avenue, Berlin, NJ 08009; Téléphone: (1)856-768-9360; Email:mjmrvica@mrvica.com.

This journal is indexed by / Cette revue est indexée par: Adis International, ArticleFirst, BIOBASE, BioLAb, BiolSci, BIOSIS Prev, Centre National de la Recherche Scientifique, CSA, CurAb, CurCont, De Gruyter Saur, E-psyche, EBSCO, Elsevier, EMBASE, FRANCIS, IBZ, Internationale Bibliographie der Rezensionen Geistes-und Sozialwissenschaftlicher Literatur, MEDLINE, MetaPress, National Library of Medicine, OCLC, PE\&ON, Personal Alert, PsycFIRST, PsycINFO, PubMed, Reac, RefZh, SCI, SCOPUS, Thomson Reuters, TOCprem, VINITI RAN, Web of Science.

ISSN: 0317-1671 EISSN: 2057-0155

COPYRIGHT ( 2016 by THE CANADIAN JOURNAL OF NEUROLOGICAL SCIENCES INC. All rights reserved. No part of this publication may be reproduced, in any form or by any means, electronic, photocopying, or otherwise, without permission in writing from Cambridge University Press. Policies, request forms and contacts are available at: http://www.cambridge.org/about-us/rights-permissions. Permission to copy (for users in the U.S.A.) is available from Copyright Clearance Center: http://www.copyright.com, email: info@ copyright.com.

COPYRIGHT () 2016 du THE CANADIAN JOURNAL OF NEUROLOGICAL SCIENCES INC. Tous droits réservés.

Aucune partie de cette publication ne peut être reproduite, sous quelque forme ou par quelque procédé que ce soit, électronique ou autre, y compris la photocopie, sans l'accord écrit de Cambridge University Press. Les politiques, les formulaires de demande et les contacts sont disponibles à: http://www.cambridge.org/aboutus/rights-permissions. La permission de copier (pour les utilisateurs aux ÉtatsUnis) est disponible auprès Copyright Clearance Center: http://www.copyright. com, email: info@copyright.com. 


\section{Abstracts}

\section{Platform Presentations}

\section{Wednesday, June 21, 2017}

GP. Grand Plenary GP.01 to GP.05

Includes all 2017 Society Prize Abstracts S7

A. CACN Chair's Select Abstract Presentations A.01 to A.07 S8
B. CNS/CSCN Chair's Select Abstract Presentations B.01 TO B.07 $\mathrm{S} 10$

C. CNSS Chair's Select Abstract Presentations C.01 to C.07 $\mathrm{S} 12$

\section{Poster Presentations}

\section{Tuesday, June 20, 2017 and Thursday, June 22, 2017}

\section{GENERAL CATEGORIES}

General Neurology - P.001 to P.005. S14

General Neurosurgery - P.006 to P.024. S15

General Pediatric Neurology - P.025 to P.026 .....S19

Pediatric Nerosurgery - P.027 to P.028 . $S 20$

Epilepsy - P.029 to P.039 $S 21$

Neuro-oncology - P.040 to P.044 . $S 24$

Neurovascular and

Neurointerventional - P.045 to P.052 $S 25$

Multidisciplinary - Other - P.053 $S 26$

Stroke - P.054 to P.063 $S 27$

\section{NEUROLOGY AND CHILD NEUROLOGY SUBSPECIALTIES}

Dementia and Cognitive

Disorders - P.064 to P.065. $S 29$

Movement Disorders - P.066.

MS / Neuroinflammatory

Disease - P.067 to P.071 S30

Neuromuscular Disease - P.072 to P.074 S30 S31
NEUROPHYSIOLOGY SUBSPECIALTIES EEG - P.075 to P.077 .......................................S32

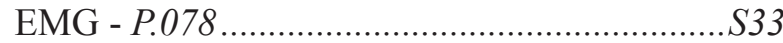

Neuroimaging - P.079 to P.083 .........................S33

NEUROSURGICAL SUBSPECIALTIES

Functional Neurosurgery and Pain - P.084 to P.085..........................................S34 Spine and Peripheral Nerve Surgery - P.086 to P.098... $S 35$

OTHER

Ethical, Legal, Societal, Historical Aspects of Neuroscience - P.099 .539

Neuroscience Education - P.100 to P.108 S39

Congress @ Glance................................................. S6

AUTHORS INDEX .......................................... S45 


\section{http://congress.cnsfederation.org/congress/societyprizes/}

\section{Society Prize Winners}

\section{Congratulations to the following CNSF members that have been awarded 2017 Society Prizes.}

\section{Be sure to attend the Grand Plenary session on Wednesday morning when they will present their work.}

The Canadian Neurological Society

2017 Francis McNaughton Memorial Prize

Recipient: $\quad$ Raed A. Joundi

Title as submitted: "Outcomes after gastrostomy tube placement in patients with acute stroke: A 10-year population-based study using the Ontario Stroke Registry"

2017 André Barbeau Memorial Prize

Recipient: $\quad$ Kristin Ikeda

Title as submitted: "Network connectivity following a single unprovoked seizure using 7 Tesla resting-state fMRI"

The Canadian Association of Child Neurology

2017 President's Prize

Recipient: $\quad$ Kristine Woodward

Title as submitted: "Sensory-motor network functional connectivity in hemiparetic children with perinatal stroke"

\section{The Canadian Neurosurgical Society}

2017 K.G. McKenzie Memorial Prize in Basic Neuroscience Research

Recipient: Daipayan Guha

Title as submitted: "Quantification of computational geometric homogeneity in surface-based registration for spinal intra-operative three dimensional navigation"

2017 K.G. McKenzie Memorial Prize in Clinical Research

Recipient: Stefan Lang

Title as submitted: "Resting state functional connectivity: a biomarker for pre-operative cognitive function and cognitive outcome following surgery in patients with diffuse glioma" 